\title{
Online-Statistiken der WIRTSCHAFTSINFORMATIK / Business \& Information Systems Engineering (BISE)
}

\section{DOI 10.1007/s11576-010-0227-1}

\section{Der Autor}

Prof. Dr. Hans Ulrich Buhl ( $\varangle)$

FIM Kernkompetenzzentrum Finanz-

\& Informationsmanagement

Universität Augsburg

Universitätsstraße 12

86159 Augsburg

Deutschland

hans-ulrich.buhl@

wiwi.uni-augsburg.de

Online publiziert: 2010-06-24

This article is also available in English via http://www.springerlink.com and http://www.bise-journal.org: Buhl HU (2010) Online statistics of Business \& Information Systems Engineering (BISE) / WIRTSCHAFTSINFORMATIK. Bus Inf Syst Eng. doi: 10.1007/s12599-0100108-6.

(C) Gabler Verlag 2010
Die WIRTSCHAFTSINFORMATIK und BISE stehen ebenfalls über http://www. wirtschaftsinformatik.de bzw. http://www.bise-journal.org zur Verfügung. Darüber hinaus sind alle seit 2006 veröffentlichten Artikel der WIRTSCHAFTSINFORMATIK sowie alle seit 2009 veröffentlichten Artikel der BISE über SpringerLink verfügbar. Das bedeutet, dass alle Personen weltweit Zugriff zu unseren Artikeln haben, welche einer Institution mit entsprechender Lizenz angehören. In den meisten Ländern gilt dies beispielsweise für nahezu alle Universitäten (und damit deren Mitarbeiter und Studierende). Damit haben international fast alle interessierten Leser die Möglichkeit, die in der WIRTSCHAFTSINFORMATIK bzw. BISE veröffentlichten Artikel zu lesen.

Hinsichtlich der internationalen Marktdurchdringung können wir in den letzten Jahren eine sehr erfreuliche Entwicklung verzeichnen: Im Gesamtjahr 2009 lag die Anzahl der Downloads, welche über SpringerLink getätigt wurden, bei 37.493 für die WIRTSCHAFTSINFORMATIK und - für eine 2009 gestartete Zeitschrift ganz außergewöhnlich - bei 6.336 für die BISE.

Im ersten Quartal 2010 wuchsen die Downloads der WIRTSCHAFTSINFORMATIK auf 9.900 (+28\% im Vergleich zum Vorjahresquartal) weiter, nachdem diese von 2007 bis 2009 bereits um ca. $745 \%$ in zwei Jahren auf ein sehr hohes Niveau angestiegen waren. Bei der englischsprachigen, neuen BISE explodierten die Downloads über SpringerLink im ersten Quartal 2010 geradezu mit einer Steigerung um über $600 \%$ im Vergleich zum Vorjahresquartal - also innerhalb eines Jahres - auf einen Wert von 4.169 Downloads. Die BISE hat bereits zu Beginn ihres zweiten Jahres $40 \%$ des - ebenfalls sehr guten - Wertes der WIRTSCHAFTSINFORMATIK mit ihrer 50-jährigen Tradition übertroffen.

Damit baut die WIRTSCHAFTSINFORMATIK im deutschsprachigen Raum ihren Vorsprung vor anderen - sehr viel breiter aufgestellten - wissenschaftlichen Zeitschriften weiter aus. Und die Internationalisierungsstrategie startete mit der BISE, welche in ihrem ersten Jahr bereits zu mehr als einem Drittel aus dem asiatisch-pazifischen Raum bezogen wurde, weit besser als das selbst die größten Optimisten unter uns erwarten konnten.

Zusammen genommen nehmen - wiederum gemessen an den Downloads - die beiden Zeitschriften bereits weltweit den dritten Platz unter allen in SpringerLink verfügbaren IS-Zeitschriften ein.

Aus diesem Anlass möchte ich mich an dieser Stelle nochmals ganz herzlich bei allen Autoren, Gutachtern und Lesern bedanken. Hierbei möchte ich neben der Leistung der Autoren auch die Tätigkeit unserer Gutachter hervorheben, die - mit in der Regel sehr kurzen Begutachtungszeiten - dafür Sorge tragen, dass wir in intensiver Zusammenarbeit mit den Autoren meist über mehrere Iterationen qualitativ hochwertige Forschungsergebnisse erhalten und präsentieren können. Vor allem aber danke ich natürlich Ihnen, liebe Leser, dass Sie in einem solchen Ausmaß zum stark wachsenden Erfolg der Zeitschrift beitragen.

Prof. Dr. Hans Ulrich Buhl Geschäftsführender Herausgeber 